\title{
Sobrevivência a Médio Prazo e Estado Funcional de Pacientes com Estenose Valvar Aórtica Grave Submetidos a Implante Transcateter da Válvula Aórtica
}

\author{
Alfonsina Candiello ${ }^{1}$, Fernando Cura ${ }^{2}$, Mariano Albertal ${ }^{3}$, Lucio T. Padilla ${ }^{4}$, Gerardo $\mathrm{Nau}^{5}$, \\ Florencia Castro ${ }^{6}$, Ricardo Ronderos ${ }^{7}$, Gustavo Avegliano ${ }^{8}$, Daniel Navia ${ }^{9}$, Jorge Belardi ${ }^{10}$
}

\section{RESUMO}

Introdução: O procedimento de implante transcateter da válvula aórtica (TAVI, do inglês transcatheter aortic valve implantation) representa opção de tratamento em pacientes com estenose valvar aórtica (EA) com risco cirúrgico elevado ou proibitivo. Relatamos nossa experiência usando o sistema autoexpansível Medtronic CoreValve ${ }^{\circledR}$. Métodos: No período de 2009 a 2013, 51 pacientes consecutivos com EA grave sintomática e risco cirúrgico alto ou proibitivo foram submetidos ao TAVI. Os resultados foram analisados de acordo com os critérios Valve Academic Research Consortium (VARC) -2. Resultados: A média de idades dos pacientes foi $82 \pm 6$ anos, $49 \%$ eram do sexo feminino, $19 \%$ diabéticos, $21 \%$ tinham insuficiência renal e o EuroSCORE logístico foi $17,4 \pm 11,4 \%$. O sucesso do dispositivo foi alcançado em $84,3 \%$. Todos os pacientes tiveram diminuição significativa dos gradientes transaórticos, que foi mantida ao longo do tempo. A internação hospitalar foi de 6 dias (intervalo interquartil: 5-8,8). A mortalidade intra-hospitalar e aos 30 dias foi $7,8 \%$ e $9,8 \%$, respectivamente. Implante de marca-passo permanente foi necessário em 32,6\% dos casos; acidente cerebrovascular isquêmico ocorreu em 3,9\%; e complicações vasculares maiores em $6 \%$ dos pacientes. A sobrevivência aos 6 meses e em 1 ano foi $86,3 \%$ e $84,4 \%$, respectivamente. A classe funcional NYHA melhorou significativamente após o TAVI e permaneceu baixa no seguimento de médio prazo. Conclusões: Nesta experiência preliminar, o tratamento de pacientes com EA e risco cirúrgico alto ou proibitivo com TAVI, usando o

\section{ABSTRACT}

Medium-Term Survival and Functional Status
of Patients With Severe Aortic Valve Stenosis
Undergoing Transcatheter Aortic Valve Implantation
Background: Transcatheter aortic valve implantation (TAVI)
is a treatment option for patients with aortic valve stenosis
(AS) and high or prohibitive surgical risk. We report our
experience using the Medtronic CoreValve ${ }^{T M}$ self-expending
system. Methods: From 2009 to 2013,51 consecutive patients
with severe symptomatic AS and high or prohibitive surgical
risk were submitted to TAVI. Results were analyzed according
to the criteria of the Valve Academic Research Consortium
(VARC) - 2 . Results: Mean age was $82 \pm 6$ years, $49 \%$ were
female, $19 \%$ were diabetic, $21 \%$ had renal failure and the
logistic EuroScore was $17.4 \pm 11.4 \%$. The success rate of
the device was $84.3 \%$. All of the patients had a significant
decrease of transaortic gradients, which was maintained over
time. Hospitalization time was 6 days (interquartile range:
$5-8,8$ ). In-hospital mortality at 30 days was $7.8 \%$ and $9.8 \%$,
respectively. Permanent pacemaker implantation was required
in $32.6 \%$ of the cases, ischemic stroke was observed in $3.9 \%$
and major vascular complications in $6 \%$ of the patients. Survival
at 6 months and 1 year was $86.3 \%$ and $84.4 \%$, respectively.
NYHA functional class improved significantly after TAVI and
remained low in the medium-term follow-up. Conclusions:
In this preliminary experience, the treatment of patients with

1 Médica do Departamento de Cardiologia Intervencionista e de Terapêutica Endovascular do Instituto Cardiovascular de Buenos Aires. Buenos Aires, Argentina.

${ }^{2}$ Chefe do Departamento de Cardiologia Intervencionista e de Terapêutica Endovascular do Instituto Cardiovascular de Buenos Aires. Buenos Aires, Argentina.

${ }^{3}$ Médico do Departamento de Cardiologia Intervencionista e de Terapêutica Endovascular do Instituto Cardiovascular de Buenos Aires. Buenos Aires, Argentina.

4 Subchefe do Departamento de Cardiologia Intervencionista e de Terapêutica Endovascular do Instituto Cardiovascular de Buenos Aires. Buenos Aires, Argentina.

${ }^{5}$ Médico do Departamento de Cardiologia Intervencionista e de Terapêutica Endovascular do Instituto Cardiovascular de Buenos Aires. Buenos Aires, Argentina.

${ }^{6}$ Médica do Departamento de Ecocardiografia do Instituto Cardiovascular de Buenos Aires. Buenos Aires, Argentina.

\footnotetext{
${ }^{7}$ Chefe do Departamento de Ecocardiografia do Instituto Cardiovascular de Buenos Aires. Buenos Aires, Argentina.

${ }^{8}$ Chefe do Departamento de Ecocardiografia do Instituto Cardiovascular de Buenos Aires. Buenos Aires, Argentina.

${ }^{9}$ Diretor do Departamento de Cirurgia Cardíaca do Instituto Cardiovascular de Buenos Aires. Buenos Aires, Argentina.

${ }^{10}$ Diretor do Departamento de Cardiologia Intervencionista e de Terapêutica Endovascular do Instituto Cardiovascular de Buenos Aires. Buenos Aires, Argentina.

Correspondência: Alfonsina Candiello. Departamento de Cardiologia Intervencionista e de Tratamento Endovascular do Instituto Cardiovascular de Buenos Aires - Blanco Encalada, 1.543, 4o andar - Cidade Autônoma de Buenos Aires, Argentina.

E-mail: alfonsinacandiello@gmail.com

Recebido em: 8/9/2013 • Aceito em: 16/11/2013
} 
sistema autoexpansível CoreValve ${ }^{\circledR}$, foi considerado viável e seguro, e levou à melhoria sustentável dos sintomas cardíacos. Após a superação dos riscos iniciais de morte e de acidente cerebrovascular, o procedimento garantiu um bom resultado clínico, no longo prazo.

DESCRITORES: Estenose da valva aórtica. Implante de prótese de valva cardíaca. Resultado de tratamento.

A estenose valvar aórtica (EA) calcificada constitui problema cardíaco comum e é particularmente prevalente na população idosa. Durante várias décadas, a substituição cirúrgica da válvula aórtica tem sido o esteio do tratamento da EA sintomática, considerada uma recomendação Classe I pelas diretrizes internacionais. ${ }^{1}$ Em indivíduos selecionados, o procedimento cirúrgico traz considerável melhora dos sintomas e da expectativa de vida. ${ }^{1}$ Não obstante, há uma considerável proporção de indivíduos idosos com EA aos quais não é oferecida a alternativa cirúrgica, em virtude do risco alto ou proibitivo. ${ }^{1}$

Por causa da necessidade não suprida para o tratamento desse subgrupo de alto risco, começaram a surgir procedimentos minimamente invasivos. O implante transcateter da válvula aórtica (TAVI) é um procedimento percutâneo, que representa uma opção de tratamento para pacientes com EA sem possibilidade cirúrgica. ${ }^{2}$

Desde o primeiro TAVI, em 2002, mais de 70 mil procedimentos foram realizados, com uma alta taxa de sucesso. No entanto, os dados demográficos de base e as características do procedimento, bem como os resultados clínicos, podem diferir em nossa região quando comparados com os verificados no resto do mundo. Até a presente data, há dados limitados em relação à viabilidade e à segurança do TAVI em nossa região. . $^{3-5}$ Desse modo, apresentamos nossa experiência inicial com o TAVI, usando o sistema autoexpansível Medtronic CoreValve $^{\circledR}$ (Medtronic Inc., Minneapolis, Estados Unidos).

\section{MÉTODOS}

\section{População de pacientes e procedimentos diagnósticos}

Entre março de 2009 e março de 2013, 51 pacientes consecutivos com EA sintomática grave e risco cirúrgico alto ou proibitivo foram submetidos ao TAVI em nossa instituição.

Uma equipe multidisciplinar determinou a elegibilidade para o TAVI com base na situação clínica e na ecocardiografia transesofágica (ETE), na angiografia coronária seletiva, na aortografia e na angiografia iliofemoral. A ETE foi realizada em todos os pacientes, enquanto que a tomografia computadorizada com múltiplos detectores (TCMD) foi realizada em apenas alguns, para avaliar a
AS and high or prohibitive surgical risk with TAVI, using the CoreValve ${ }^{\mathrm{TM}}$ self-expanding system was feasible and safe and led to sustained improvement of cardiac symptoms. After overcoming the initial risks of death and stroke, the procedure guaranteed good long-term clinical outcomes.

KEYWORDS: Aortic valve stenosis. Heart valve prosthesis implantation. Treatment outcome.

anatomia da raiz aórtica (incluindo a avaliação do anel aórtico) e a viabilidade da abordagem transfemoral.

EA grave foi definida como uma área da válvula aórtica $<0,8 \mathrm{~cm}^{2}$, além de gradiente médio da válvula aórtica $>40 \mathrm{mmHg}$, ou velocidade máxima do jato aórtico > 4,0 m/s.

Os pacientes foram considerados como de alto risco de complicações cirúrgicas ou de morte com base nas condições coexistentes associadas ao risco de morte de, pelo menos, $15 \%$ aos 30 dias após o procedimento (escore Society of Thoracic Surgeons - STS $\geq 10$ ou EuroScore logístico $\geq 15$ ).

Os critérios anatômicos de inclusão foram os seguintes: orifício da válvula aórtica com área $<0,8 \mathrm{~cm}^{2}$, diâmetro do anel aórtico $\geq 20 \mathrm{~mm}$ e $\leq 29 \mathrm{~mm}$, e diâmetro da aorta ascendente ao nível da junção sinotubular $\leq 45 \mathrm{~mm}$.

Os pacientes foram excluídos se houvesse trombo intracavitário; fração de ejeção do ventrículo esquerdo (FEVE) extremamente baixa $(<20 \%)$ e irreversível; regurgitação mitral ou aórtica grave (4+); e eixo arterial iliofemoral com calibre $<6 \mathrm{~mm}$ ou com tortuosidade grave, que pudesse impedir a entrega da prótese ou implante do óstio coronário baixo (<10 mm).

O sucesso do dispositivo foi definido como ausência de mortalidade no procedimento e posicionamento correto de uma única prótese na localização apropriada, e com desempenho adequado (sem mismatch prótese-paciente; gradiente médio da válvula aórtica $<20 \mathrm{mmHg}$, ou velocidade máxima < $3 \mathrm{~m} / \mathrm{s}$; e sem regurgitação moderada/grave da prótese valvular).

Todos os pacientes forneceram consentimento informado para serem submetidos ao TAVI pela via transfemoral, incluindo consentimento para o processamento anônimo de seus dados. A informação sobre morte foi obtida no hospital ou por contato telefônico com a família do paciente. Todos os eventos foram definidos de acordo com os critérios Valve Academic Research Consortium (VARC) $-2 .{ }^{6}$

\section{Procedimento de implantação}

Todos os procedimentos foram realizados sob anestesia geral, utilizando a prótese autoexpansível CoreValve ${ }^{\circledR}$ (Medtronic, Minneapolis, Minnesota, Estados Unidos) 18 F. Brevemente, a prótese CoreValve ${ }^{\circledR}$ consiste em 
tecido pericárdico porcino montado num stent autoexpansível de nitinol.

Os procedimentos foram inicialmente realizados com um cateter de liberação $18 \mathrm{~F}$ que, mais tarde, foi aprimorado por uma membrana estabilizadora AccuTrak ${ }^{\circledR}$. Três tamanhos de próteses estavam disponíveis (26, 29 e $31 \mathrm{~mm}$ ) para os tamanhos do anel da válvula aórtica 20 e $29 \mathrm{~mm}$. Detalhes do dispositivo e dos aspectos técnicos do procedimento foram previamente publicados. $^{7}$

O acesso transarterial foi obtido por via percutânea ou por incisão cirúrgica. Após obter acesso vascular, um fio-guia de 0,35" (um fio J normal ou um fio reto, quando apropriado) foi posicionado no ventrículo esquerdo e dois pigtails foram colocados na aorta ascendente e no ventrículo esquerdo, para realizar medidas simultâneas de pressão e avaliação do gradiente transvalvar. O fio-guia intraventricular foi, então, trocado por um fio de alto suporte, com curva feita sob medida, e posicionado no ápice. O posicionamento e o implante da prótese valvular no anel aórtico foram guiados por fluorocospia e por ETE. A pré-dilatação foi deixada a critério do operador. A liberação gradual da prótese foi feita pela retração da bainha. Pós-dilatação foi realizada nos casos de subexpansão do dispositivo. O acesso femoral foi fechado cirurgicamente ou percutaneamente com dois Perclose ProGlide $^{\circledR} 6$ F Suture-Mediated Closure System (Abbott Vascular, Redwood City, Estados Unidos).

\section{Medicação}

Todos os pacientes receberam ácido acetilsalicílico antes do procedimento e continuaram a recebê-lo indefinidamente. Foi administrada a eles também uma dose de ataque de clopidogrel de $300 \mathrm{mg}$. A dose de manutenção de clopidogrel, administrada durante 3 meses, foi $75 \mathrm{mg}$. Durante o procedimento, heparina não fracionada foi administrada de acordo com o peso do paciente, para atingir um tempo de coagulação ativada $\geq 250$ segundos.

\section{Seguimento}

Exames clínico e ecocardiográfico foram realizados antes da alta hospitalar, aos 30 dias, 6 e 12 meses. Os resultados foram analisados de acordo com desfechos padrão atualizados, definidos pelos critérios VARC-2.

\section{Análise estatística}

As variáveis qualitativas foram expressas como porcentagens e as quantitativas como média \pm desvio padrão, ou pela mediana (intervalo interquartil - IQ, $25 \%$ a $75 \%$ ). As variáveis categóricas foram comparadas com o teste qui-quadrado. Para a comparação entre os gradientes seriados médio e máximo, foi usado o teste $t$ pareado. A análise estatística foi realizada usando o Statistical Package for the Social Sciences (SPSS) versão 21.0 (IBM, Armonk, Nova Iorque, Estados Unidos).

\section{RESULTADOS}

\section{Características dos pacientes}

As características basais estão resumidas na Tabela 1. Todos os pacientes tinham dispneia, a maioria deles em repouso ou após atividade física mínima. A idade média foi $82 \pm 6$ anos, $49 \%$ deles eram do sexo feminino, 19\% eram diabéticos e 21\% tinham insuficiência renal crônica. A média do EuroSCORE logístico foi 17,4 \pm $11,4 \%$ e $17 \%$ dos pacientes apresentaram comorbidades que não são medidas nos atuais escores de riscos cirúrgicos: quatro pacientes $(7,8 \%)$ tinham aorta em porcelana, três pacientes $(5,8 \%)$ apresentaram deformidade grave da parede torácica ou irradiação do tórax prévia e um paciente realizou transplante hepático prévio em terapêutica imunossupressora. A Tabela 1 mostra os parâmetros ecocardiográficos basais, que ilustram a gravidade da EA.

TABELA 1

Características dos pacientes $(n=51)$

\begin{tabular}{|c|c|}
\hline Idade, anos & $82 \pm 5,7$ \\
\hline Sexo feminino, $\mathrm{n}(\%)$ & $25(49)$ \\
\hline Índice de massa corporal, kg/m² & $27 \pm 4$ \\
\hline EuroSCORE logístico, \% & $17,4 \pm 11,7$ \\
\hline Escore STS*, \% & $10,2 \pm 9$ \\
\hline \multicolumn{2}{|l|}{ Classe funcional $N Y H A^{\star \star}, \mathrm{n}(\%)$} \\
\hline$\|$ & $6(11,8)$ \\
\hline III & $18(35,3)$ \\
\hline IV & $27(52,9)$ \\
\hline Hipertensão arterial, n (\%) & $43(84,3)$ \\
\hline Dislipidemia, n (\%) & $35(68,6)$ \\
\hline Diabetes mellitus, $\mathrm{n}(\%)$ & $10(19,6)$ \\
\hline Tabagismo, n (\%) & $15(29,4)$ \\
\hline Acidente cerebrovascular prévio, n (\%) & $4(7,8)$ \\
\hline Fibrilação atrial, n (\%) & $11(21,6)$ \\
\hline Marca-passo permanente, $\mathrm{n}(\%)$ & $6(11,8)$ \\
\hline Angioplastia prévia, n (\%) & $16(31,4)$ \\
\hline Cirurgia cardíaca prévia, n (\%) & $8(15,7)$ \\
\hline Doença pulmonar obstrutiva crônica, n (\%) & $5(9,8)$ \\
\hline Insuficiência renal crônica, n (\%) & $11(21,6)$ \\
\hline Doença arterial periférica, $\mathrm{n}(\%)$ & $11(21,6)$ \\
\hline Fragilidade, $\mathrm{n}(\%)$ & $17(33,3)$ \\
\hline \multicolumn{2}{|l|}{ Achados ecocardiográficos } \\
\hline Área da válvula aórtica, $\mathrm{cm}^{2}$ & $0,5 \pm 0,2$ \\
\hline Gradiente médio da válvula aórtica, mmHg & $50 \pm 10$ \\
\hline Fração de ejeção do ventrículo esquerdo, \% & $55 \pm 13$ \\
\hline Tamanho do anel aórtico, $\mathrm{cm}^{2}$ & $23 \pm 2$ \\
\hline \multicolumn{2}{|c|}{$\begin{array}{l}\text { * STS: Society of Thoracic Surgeons. } 0 \text { escore STS mede o risco no momento da } \\
\text { cirurgia cardiovascular numa escala que vai de zero a cem. Um número na escala } \\
\text { superior a } 10 \% \text { indica risco cirúrgico muito alto; }{ }^{*} \text { classe funcional NYHA: Classe } \\
\text { funcional New York Heart Association. }\end{array}$} \\
\hline
\end{tabular}




\section{Procedimento}

De um total de 51 pacientes, 82,4\% realizaram procedimentos eletivos e os demais, urgentes. O acesso percutâneo total foi realizado em $43 \%$ dos casos, e a maioria dos procedimentos (50/51) foi guiada por ETE (Tabela 2).

Foram implantados dispositivos CoreValve ${ }^{\circledR}$ de 26 e de $29 \mathrm{~mm}$ em $44 \%$ e em $52 \%$ dos pacientes, respectivamente. O dispositivo CoreValve ${ }^{\circledR}$ de $31 \mathrm{~mm}$ passou a estar disponível a partir de dezembro de 2012 e foi usado em dois casos (4\%). Pré-dilatação com balão foi realizada em 28 pacientes (54,9\%), enquanto que 19 deles $(37,3 \%)$ necessitaram de pós-dilatação, em virtude de subexpansão da válvula imediatamente após o implante valvular.

O sucesso do dispositivo ocorreu em $84,3 \%$ dos pacientes (43/51). Foi observada uma dramática diminuição dos gradientes transvalvares máximos (de $83 \pm 22$ para $13 \pm 5 \mathrm{mmHg} ; \mathrm{p}<0,001$ ) e médio (de $50 \pm 10$ para 6,4 $\pm 2,6 \mathrm{mmHg} ; \mathrm{p}<0,001)$ pela ecocardiografia, imediatamente após o procedimento. No final do procedimento, nenhum paciente teve regurgitação

TABELA 2

Resultados do procedimento e seguimento $(n=51)$

\begin{tabular}{lc}
\hline Resultados & \\
\hline Procedimento, $\mathrm{n}(\%)$ & $42(82,4)$ \\
Eletivo & $9(17,6)$ \\
Urgente & \\
Tamanho rotulado da prótese em mm, $\mathrm{n}(\%)$ & $22(43,1)$ \\
26 & $26(51,0)$ \\
29 & $2(3,9)$ \\
31 & $51(100)$ \\
Anestesia geral, $\mathrm{n}(\%)$ & $50(98,0)$ \\
Ecocardiografia transesofágica, $\mathrm{n}(\%)$ & $22(43,1)$ \\
Procedimento totalmente percutâneo, $\mathrm{n}(\%)$ & $28(54,9)$ \\
Pré-dilatação, $\mathrm{n}(\%)$ & $19(37,3)$ \\
Pós-dilatação, $\mathrm{n}$ (\%) & $43(84,3)$ \\
Sucesso do dispositivo*, $\mathrm{n}(\%)$ & \\
Morte, $\mathrm{n}$ (\%) & $2(3,9)$ \\
$\quad$ Intraprocedimento & $4(7,8)$ \\
$\quad$ Intra-hospitalar & $5(9,8)$ \\
30 dias & $7(13,7)$ \\
6 meses & $8(15,7)$ \\
1 ano & \\
Regurgitação aórtica, $\mathrm{n}(\%)$ & $6(11,6)$ \\
$\geq$ grau 2 pós-procedimento & $5(9,8)$ \\
$\geq$ grau 2 ao final de 1 ano & $2(3,9)$ \\
\hline * Sucesso do dispositivo é definido como implante da prótese sem regurgitação \\
aórtica moderada/grave ou morte. \\
\hline
\end{tabular}

aórtica grave; 6 pacientes $(11,8 \%)$ tiveram regurgitação aórtica moderada e 32 pacientes $(62,7 \%)$, regurgitação aórtica leve.

Dois pacientes morreram durante o procedimento (um deles por causa de ruptura do anel durante a pré-dilatação e o outro imediatamente antes da prédilatação, em virtude de instabilidade hemodinâmica secundária). Dois pacientes precisaram de uma segunda válvula (um deles por causa de embolização da prótese no arco aórtico e o outro devido ao implante anular profundo associado à regurgitação aórtica grave).

\section{Resultados intra-hospitalar e aos 30 dias}

A mediana da internação hospitalar foi de 6 dias $(\mathrm{IQ}=5-8,8)$ e $32,6 \%$ dos pacientes receberam um marca-passo permanente. A mortalidade intra-hospitalar e aos 30 dias, por todas as causas, foi $7,8 \%(4 / 51)$ e $9,8 \%(5 / 51)$, respectivamente. Dois pacientes $(3,9 \%)$ tiveram acidente cerebrovascular isquêmico. Grandes complicações vasculares ocorreram em três pacientes $(5,9 \%)$.

\section{Seguimento}

A redução inicial observada dos gradientes máximo e médio, após o procedimento, foi mantida durante todo o seguimento (Figura 1).

A sobrevivência aos 6 meses e em 1 ano foi $86,3 \%$ e $84,4 \%$, respectivamente. A classe funcional NYHA melhorou significativamente após o TAVI e permaneceu baixa no seguimento de longo prazo (Figura 2). Em 1 ano, nenhum paciente teve regurgitação aórtica grave, enquanto que 5/43 pacientes $(11,6 \%)$ tiveram regurgitação aórtica moderada.

\section{DISCUSSÃO}

A EA sintomática é doença cardíaca associada a um estado funcional debilitado e que prenuncia um mal prognóstico. ${ }^{1}$ Durante décadas, os indivíduos idosos com essa doença não receberam, de modo geral, o tratamento adequado, em razão da presença

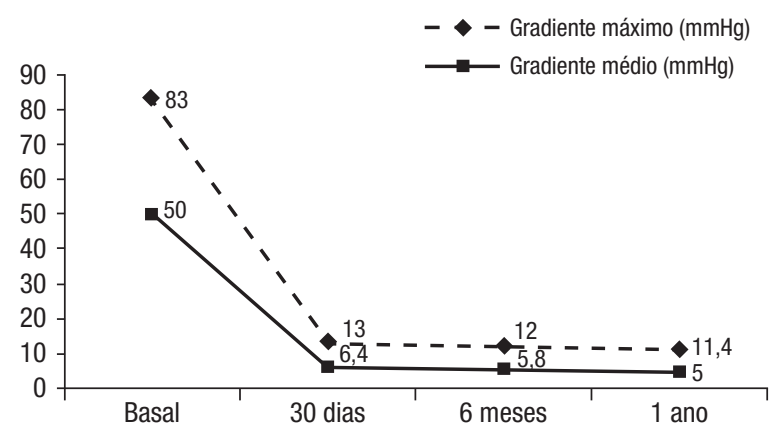

Figura 1. Avaliação por ecocardiografia seriada dos gradientes aórticos transvalvulares médio e máximo basais, aos 30 dias, 6 meses e 1 ano de seguimento. 


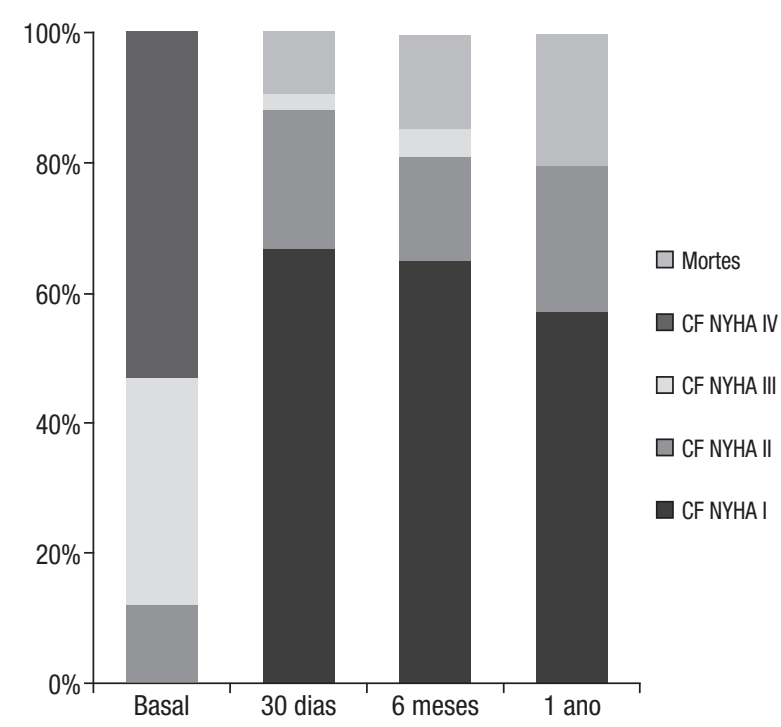

Figura 2. Taxa de mortalidade e classe funcional NYHA aos 30 dias, 6 meses e 1 ano de seguimento. CF NYHA = Classe funcional New York Heart Association.

de comorbidades e do elevado risco cirúrgico. Relatamos aqui nossa experiência inicial com o TAVI, um procedimento alternativo minimamente invasivo para o tratamento definitivo de pacientes com EA sintomática que apresentam risco cirúrgico alto ou proibitivo.

No presente estudo, a mortalidade aos 30 dias foi $9,8 \%$, o que está de acordo com outros registros de TAVI que usaram sistemas CoreValve ${ }^{\circledR}$ (UK Registry: 7,1\%; French Registry: 12,7\%; France-2 Registry: 9,4\% e German Registry: $12,4 \%$ ), ${ }^{8-10}$ refletindo a viabilidade e a segurança do procedimento na população de pacientes da prática clínica com EA, que geralmente permanece sem o tratamento adequado. A taxa de mortalidade do presente estudo aos 30 dias foi levemente superior à observada num estudo brasileiro recentemente publicado (6,7\%). Diferentemente do nosso estudo, os autores realizaram o TAVI sem pré-dilatação com balão, o que pode ter ajudado a melhorar a sobrevivência a curto prazo. ${ }^{4} \mathrm{Em}$ seguida, um registro TAVI brasileiro ( $n=112$ ), com pacientes submetidos a uma pré-dilatação obrigatória, mostrou uma mortalidade semelhante $(9,4 \%){ }^{3}$ Também em linha com registros anteriores, 8,11 a sobrevivência em 1 ano foi de 84,4\%. Nós também verificamos uma queda significativa do gradiente da válvula aórtica, que se traduziu numa diminuição sustentada dos sintomas cardíacos após o TAVI. Portanto, após uma exposição inicial a um risco, os pacientes que sobreviveram tiveram uma melhora considerável da Qualidade de Vida e um excelente resultado clínico no longo prazo, confirmando a durabilidade do dispositivo.

No nosso estudo, o risco cirúrgico elevado (EuroSCORE logístico: $17,4 \pm 11,4 \%$ ) teria contraindicado a subs- tituição cirúrgica da válvula ou levado à mortalidade e à morbidade excessivas, consequentes à cirurgia. Parece que o risco do TAVI é razoável, em razão da falta de um tratamento alternativo definitivo e do mau prognóstico observado com o tratamento somente medicamentoso. Conforme mostraram registros contemporâneos do TAVI, ${ }^{12-15}$ os eventos adversos cardiovasculares diminuem com os avanços na tecnologia dos dispositivos e a crescente experiência dos operadores e do heart team.

Há considerável evidência indicando que a regurgitação aórtica paravalvular, após o TAVI, pode levar a um prognóstico pior. ${ }^{16,17}$ De acordo com séries anteriores, a regurgitação aórtica paravalvular após o TAVI está presente em 48 a 93\% dos casos, ${ }^{18,19}$ enquanto que a taxa de regurgitação aórtica moderada fica ao redor de 14 a $21 \% .{ }^{18,19}$ Em linha com esses estudos, 12,2\% dos nossos pacientes tiveram regurgitação aórtica moderada após o TAVI. Da mesma forma, uma experiência brasileira em 112 pacientes demonstrou taxa de regurgitação aórtica moderada/grave pós-procedimento de $11,6 \%{ }^{3}$ No início do TAVI, a maioria dos centros realizou avaliações basais pré-procedimento com eco bidimensional; no entanto, vários estudos demonstraram posteriormente que o anel aórtico frequentemente é uma estrutura elíptica e que, consequentemente, a avaliação ecocardiográfica bidimensional é imprecisa, porque ela assume que o anel é circular. Estudos mais recentes mostraram que uma avaliação mais precisa do anel aórtico permite fazer uma melhor seleção do tamanho da prótese, o que se traduz num menor vazamento paravalvular. ${ }^{20,21}$ Consequentemente, as diretrizes atuais indicam que a avaliação pré-procedimento deve ser realizada com eco tridimensional ou com TCMD, para conseguir uma medida precisa do perímetro e da área do anel. 1,20,21 Além disso, uma nova geração de próteses, com melhor vedação e capacidade de ser reposicionada e recapturada, será provavelmente capaz de diminuir o vazamento paravalvular. ${ }^{22-24}$

O implante de marca-passo continua a ser um motivo de preocupação, especialmente após o implante da CoreValve ${ }^{\circledR}$. Em linha com estudos anteriores com a CoreValve $^{\circledR}$, aproximadamente um terço dos pacientes necessita de um marca-passo permanente. ${ }^{25-28}$ Curiosamente, a necessidade de um marca-passo permanente não parece prejudicar a sobrevivência dos indivíduos submetidos ao TAVI. ${ }^{27,29}$ A presença de um novo bloqueio do ramo esquerdo e/ou de uma duração do QRS > 120 ms, após a liberação da prótese, pode prever o desenvolvimento de um bloqueio AV de alto grau e ajudar a orientar as decisões clínicas. No entanto, indicações clínicas precisas, relacionadas ao implante de marca-passo após o TAVI, ainda estão sendo desenvolvidas.

Até o momento, as taxas relatadas de acidentes cerebrovasculares isquêmicos após o TAVI têm permanecido ao redor de 1 a 10\%. ${ }^{30,31} \mathrm{Na}$ nossa série, dois pacientes 
tiveram um acidente cerebrovascular isquêmico, um deles com uma transformação hemorrágica fatal no contexto de uma síndrome de resposta inflamatória sistêmica e de trombocitopenia grave. O segundo paciente também teve uma transformação hemorrágica, mas com leve envolvimento neurológico.

\section{CONCLUSÕES}

Nesta experiência preliminar, de um único centro, o tratamento de pacientes com estenose valvar aórtica e risco cirúrgico alto ou proibitivo com o implante transcateter da válvula aórtica usando o sistema autoexpansível CoreValve ${ }^{\circledR}$ foi considerado viável e seguro, levando a uma melhora sustentável dos sintomas cardíacos. Após ultrapassar os riscos iniciais de morte e de acidente vascular cerebral, o procedimento garantiu um bom resultado clínico no longo prazo.

\section{CONFLITO DE INTERESSES}

Os autores declaram não haver conflito de interesses relacionados a este manuscrito.

\section{REFERÊNCIAS}

1. Vahanian A, Alfieri $O$, Andreotti F, Antunes MJ, BaronEsquivias G, Baumgartner $\mathrm{H}$, et al. Guidelines on the management of valvular heart disease (version 2012). Eur Heart J. 2012;33(19):2451-96.

2. Leon MB, Kodali S, Williams M, Oz M, Smith C, Stewart A, et al. Transcatheter aortic valve replacement in patients with critical aortic stenosis: Rationale, device descriptions, early clinical experiences, and perspectives. Semin Thorac Cardiovasc Surg. 2006;18(2):165-74.

3. Lluberas S, Siqueira D, Costa Jr JR, Abizaid A, Ramos A, Le Bihan D, et al. Análise da incidência e preditores clínicos e ecocardiográficos do refluxo paraprotético aórtico após o implante de prótese aórtica transcateter. Rev Bras Cardiol Invasiva. 2013;21(2):103-8.

4. Grube E, Naber C, Abizaid A, Sousa E, Mendiz O, Lemos $P$, et al. Feasibility of transcatheter aortic valve implantation without balloon pre-dilation: a pilot study. JACC Cardiovasc Interv. 2011;4(7):751-7.

5. Gaia DF, Palma JH, Ferreira CB, Souza JA, Gimenes MV, Macedo MT, et al. Transcatheter aortic valve implantation: results of the current development and implantation of a new Brazilian prosthesis. Rev Bras Cir Cardiovasc. 2011;26(3):338-47.

6. Kappetein AP, Head SJ, Genereux P, Piazza N, van Mieghem $\mathrm{NM}$, Blackstone EH, et al. Updated standardized endpoint definitions for transcatheter aortic valve implantation: the Valve Academic Research Consortium-2 consensus document (VARC-2). Eur J Cardiothoracic Surg. 2012;42(5):S45-60.

7. Buellesfeld L, Gerckens U, Schuler G, Bonan R, Kovac J, Serruys PW, et al. 2-year follow-up of patients undergoing transcatheter aortic valve implantation using a self-expanding valve prosthesis. J Am Coll Cardiol. 2011;57(16):1650-7.

8. Moat NE, Ludman $\mathrm{P}$, de Belder MA, Bridgewater B, Cunningham $A D$, Young $C P$, et al. Long-term outcomes after transcatheter aortic valve implantation in high-risk patients with severe aortic stenosis: The U.K. TAVI (United Kingdom Transcatheter Aortic Valve Implantation) registry. J Am Coll Cardiol. 2011;58(20):2130-8.

9. Eltchaninoff H, Prat A, Gilard M, Leguerrier A, Blanchard D,
Fournial G, et al. Transcatheter aortic valve implantation: early results of the FRANCE (French Aortic National Corevalve and Edwards) registry. Eur Heart J. 2011;32(2):191-7.

10. Beckmann A, Hamm C, Figulla HR, Cremer J, Kuck KH, Lange $R$, et al. The German Aortic Valve Registry (GARY): a nationwide registry for patients undergoing invasive therapy for severe aortic valve stenosis. Thorac Cardiovasc Surg. 2012; 60(5):319-25

11. Gilard $M$, Eltchaninoff $H$, lung $B$, Donzeau-Gouge $P$, Chevreul $\mathrm{K}$, Fajadet J, et al. Registry of transcatheter aortic-valve implantation in high-risk patients. N Engl J Med. 2012;366(18): 1705-15.

12. Scherner M, Madershahian N, Rosenkranz S, Slottosch I, Kuhn E, Langebartels G, et al. Transapical aortic valve implantation: experiences and survival analysis up to three years. J Cardiac Surg. 2012;27(6):653-61.

13. Hayashida K, Lefevre T, Chevalier B, Hovasse T, Romano M, Garot $\mathrm{P}$, et al. True percutaneous approach for transfemoral aortic valve implantation using the Prostar XL device: impact of learning curve on vascular complications. JACC Cardiovasc Interv. 2012;5(2):207-14.

14. Alli OO, Booker JD, Lennon RJ, Greason KL, Rihal CS, Holmes DR Jr. Transcatheter aortic valve implantation: assessing the learning curve. JACC Cardiovasc Interv. 2012;5(1):72-9.

15. Gurvitch R, Tay EL, Wijesinghe N, Ye J, Nietlispach F, Wood DA, et al. Transcatheter aortic valve implantation: lessons from the learning curve of the first 270 high-risk patients. Catheter Cardiovasc Interv. 2011;78(7):977-84.

16. Toggweiler S, Humphries KH, Lee M, Binder RK, Moss RR, Freeman $M$, et al. 5-year outcome after transcatheter aortic valve implantation. J Am Coll Cardiol. 2013;61(4):413-49.

17. Gènèreux $P$, Head $S$ J, Hahn R, Daneault $B$, Kodali $S$, Williams $M R$, et al. Paravalvular leak after transcatheter aortic valve replacement: the new Achilles heel? A comprehensive review of the literature. J Am Coll Cardiol. 2013;61(11):1125-36.

18. Patsalis PC, Konorza TF, Al-Rashid F, Plicht B, Riebisch M, Wendt $\mathrm{D}$, et al. Incidence, outcome and correlates of residual paravalvular aortic regurgitation after transcatheter aortic valve implantation and importance of haemodynamic assessment. Eurolntervention. 2013;8(12):1398-406.

19. Unbehaun A, Pasic M, Dreysse S, Drews T, Kukucka $M$, Mladenow A, et al. Transapical aortic valve implantation: incidence and predictors of paravalvular leakage and transvalvular regurgitation in a series of 358 patients. J Am Coll Cardiol. 2012;59(3):211-21.

20. Jabbour A, Ismail TF, Moat N, Gulati A, Roussin I, Alpendurada $F$, et al. Multimodality imaging in transcatheter aortic valve implantation and post-procedural aortic regurgitation: comparison among cardiovascular magnetic resonance, cardiac computed tomography, and echocardiography. J Am Coll Cardiol. 2011;58(21):2165-73.

21. Jilaihawi $H$, Kashif M, Fontana G, Furugen A, Shiota T, Friede $\mathrm{G}$, et al. Cross-sectional computed tomographic assessment improves accuracy of aortic annular sizing for transcatheter aortic valve replacement and reduces the incidence of paravalvular aortic regurgitation. J Am Coll Cardiol. 2012;59(14):1275-86.

22. Sundermann SH, Grunenfelder J, Corti R, Rastan AJ, Linke A, Lange $R$, et al. Feasibility of the EngagerTM aortic transcatheter valve system using a f lexible over-the-wire design. Eur J Cardiothorac Surg. 2012;42(4):e48-52.

23. Walther T, Thielmann $M$, Kempfert J, Schroefel H, WimmerGreinecker G, Treede H, et al. PREVAIL TRANSAPICAL: multicentre trial of transcatheter aortic valve implantation using the newly designed bioprosthesis (SAPIEN-XT) and delivery system (ASCENDRA-II). Eur J Cardiothorac Surg. 2012;42(2):278-83. 
24. Treede H, Mohr FW, Baldus S, Rastan A, Ensminger S, Arnold $M$, et al. Transapical transcatheter aortic valve implantation using the jenavalve system: acute and 30-day results of the multicentre ce-mark study. Eur J Cardiothorac Surg. 2012; 41(6):e131-8

25. van der Boon RM, Van Mieghem NM, Theuns DA, Nuis RJ, Nauta ST, Serruys PW, et al. Pacemaker dependency after transcatheter aortic valve implantation with the self-expanding Medtronic CoreValve System. Int J Cardiol. 2013;168(2):1269-73.

26. Schroeter T, Linke A, Haensig M, Merk DR, Borger MA, Mohr $F W$, et al. Predictors of permanent pacemaker implantation after Medtronic CoreValve bioprosthesis implantation. Europace. 2012;14(12):1759-63.

27. De Carlo M, Giannini C, Bedogni F, Klugmann S, Brambilla $N$, De Marco F, et al. Safety of a conservative strategy of permanent pacemaker implantation after transcatheter aortic CoreValve implantation. Am Heart J. 2012;163(3):492-9.
28. Khawaja MZ, Rajani R, Cook A, Khavandi A, Moynagh A Chowdhary $\mathrm{S}$, et al. Permanent pacemaker insertion after CoreValve transcatheter aortic valve implantation: incidence and contributing factors (the UK Corevalve Collaborative). Circulation. 2011;123(9):951-60.

29. Ledwoch J, Franke J, Gerckens U, Kuck KH, Linke A, Nickenig $\mathrm{G}$, et al. Incidence and predictors of permanent pacemaker implantation following transcatheter aortic valve implantation: analysis from the German transcatheter aortic valve interventions registry. Catheter Cardiovasc Interv. 2013;82(4):E569-77.

30. Leker RR, Eichel R, Verber A, Cohen JE, Lotan C, Danenberg HD. Stroke complicating transcatheter aortic valve implantation: Incidence, risk factors and outcome. Int J Stroke. 2013;8(4):235-9.

31. Nuis RJ, Van Mieghem NM, Schultz CJ, Moelker A, van der Boon RM, van Geuns RJ, et al. Frequency and causes of stroke during or after transcatheter aortic valve implantation. Am J Cardiol. 2012;109(11):1637-43. 